\title{
IDENTIFICAÇÃO DE SCANNERS A PARTIR DE IMAGENS/DOCUMENTOS DIGITAIS
}

\section{SCANNERS IDENTIFICATION FROM DIGITAL IMAGES/DOCUMENTS}

\author{
Gualther Hugo Aragão; Danillo Roberto Pereira; Francisco Assis da \\ Silva; Mário Augusto Pazoti; Leandro Luiz de Almeida; Helton Molina \\ Sapia; Robson Augusto Siscoutto \\ Universidade do Oeste Paulista, Faculdade de Informática de Presidente Prudente \\ E-mail: gualther-aragao@outlook.com; \\ \{danilopereira,chico,mario,llalmeida,helton,robson\}@unoeste.br
}

RESUMO - Em diversas áreas do conhecimento o uso de imagens digitais é de grande importância, e conhecer a sua origem e autenticidade é algo primordial; pois falsificações podem ser feitas facilmente. A criação de métodos que possibilitem identificar dispositivos geradores de imagens se torna fundamental. Este trabalho apresenta um ferramental matemático que identifica e classifica imagens provenientes de um determinado scanner. A construção do método foi feita utilizando a transformada wavelet e algumas técnicas de thresholding e filtragem. Paralelamente foi criada uma base de imagens digitalizadas, utilizando três modelos de scanners, para testar e validar a ferramenta desenvolvida. Os resultados dos testes realizados foram considerados satisfatórios, pois obtiveram a acurácia média de 87,5\% de acertos.

Palavras-chave: identificação de scanners; Wavelet; Thresholding; computação forense.

ABSTRACT - In several areas of knowledge about the use of digital images of great importance, and knowing their origin and authenticity is paramount; For fakes can be easily made. The creation of methods that allow the identification of imaging devices becomes fundamental. This work presents a mathematical learning that identifies and classifies images coming from a certain scanner. The construction of the method was done using a transformed wavelet and some threshold and filtering techniques. In parallel, a database of scanned images was created, using three models of scanners, to test and validate a developed tool. The results of the tests were considered satisfactory, because they obtained the accuracy average of $87.5 \%$.

Recebido em: 20/05/2016

Revisado em: 15/09/2016

Aprovado em: 03/11/2016

Keywords: Scanner Identification; Wavelet; Thresholding; computer forensics. 


\section{INTRODUÇÃO}

Com a disseminação de dispositivos tecnológicos como computadores, smartphones, câmeras digitais, scanners e internet, fez com que o volume do compartilhamento de informações aumentasse consideravelmente. Tal fato, trouxe novas modalidades de crimes, como fraudes bancárias, roubos de informações, adulteração e manipulação de imagens e documentos.

Assim surgiu a necessidade de criar um novo campo de trabalho chamado Ciência Forense Computacional. Segundo Gomes (2012) tem por objetivo estudar formas de adquirir, preservar, recuperar e analisar dados em formato eletrônico de algum tipo de mídia computacional para verificar se houve manipulação nas evidências coletadas em determinada investigação.

Imagens são de suma importância, em várias áreas do conhecimento. Algumas aplicações requerem conhecer a origem e autenticidade de imagens. Para isso é importante que métodos de identificação de dispositivos geradores de imagens sejam utilizados ou desenvolvidos.

A identificação da origem de uma imagem ou documento possibilita, saber o arquivo em questão trata-se de uma possível falsificação. Tal tarefa pode ser realizada por meio de diversos métodos.
O presente trabalho apresenta um ferramental matemático, baseado em wavelet que possibilita a identificação e classificação de imagens provenientes de um dado scanner. Um objetivo secundário a criação de uma base de imagens digitalizadas por três diferentes scanners, que além de possibilitar o teste e a validação do método desenvolvido. Também servirá de fonte para outras pesquisas.

O restante está organizado do seguinte modo: A Seção 2 aborda conceitos sobre a transformada wavelet e as técnicas de thresholding. A Seção 3 apresenta a metodologia utilizada no desenvolvimento da ferramenta e na criação da base de imagens. A Seção 4 discute os experimentos realizados e seus resultados. A Seção 5 aborda as conclusões e reflete sobre a necessidade de melhorias a serem feitas.

\section{FUNDAMENTAÇÃO TEÓRICA}

\subsection{Wavelet}

As transformadas wavelet são uma forma de se decompor um sinal em diversas partes, permitindo assim realizar uma análise em diferentes frequências e resoluções (LIMA, 2003).

Este tipo de transformada por muito tempo ficou sem uma utilização prática, a única base ortonormal conhecida eram as wavelets de Haar. Posteriormente, surgiram 
novas bases a partir de estudos de métodos de processamento digital de imagens (LIMA, 2003).

Existem dois tipos básicos de transformada: (i) contínua e (ii) discreta. $\mathrm{Na}$ transformada contínua (Equação 1) os cálculos dos coeficientes exigem grande esforço computacional e podem gerar informações redundantes. A implementação digital da transformada wavelet requer o uso de intervalos discretos entre $b$ e $a$. (FARIA, 1997; SANTOS, 2004).

$W_{\psi} f(b, a):=\left\langle f(t), \psi_{b, a}(t)\right\rangle=\int_{-\infty}^{\infty} f(t) \overline{\psi_{b, a}}(t) d t$

A discretização de $b$ deve ser tal que wavelets estreitas (de alta frequência) sejam deslocados por passos pequenos e wavelets largas (de baixa frequência) sejam deslocados por passos maiores. Com a discretização da equação №1 obtêm-se a seguinte aproximação (ARÊDES, 2009):

$$
W_{\psi} f\left(k 2^{-s}, 2^{-s}\right) \approx 2^{\frac{s}{2}} \sum_{n} f(n) \psi\left(2^{s} n-k\right)
$$

Para a aplicação de transformada wavelet discreta (DWT), é necessário o uso de bases ortogonais. Neste trabalho foi aplicada a base Wavelet de Haar, que se encontra definida a seguir:

$$
\psi(t)=\left\{\begin{array}{c}
1 \text { para } 0 \leq t \leq \frac{1}{2} \\
-1 \text { para } \frac{1}{2} \leq t<1 \\
0, \text { caso contrario }
\end{array}\right\}
$$

\subsubsection{Wavelet Bidimensional}

Para estender o uso da transformada wavelet unidimensional, existem duas formas a decomposição padrão e a decomposição não-padrão, que são visualizadas na Figura 1.

Figura 1. (a) Decomposição padrão, (b)

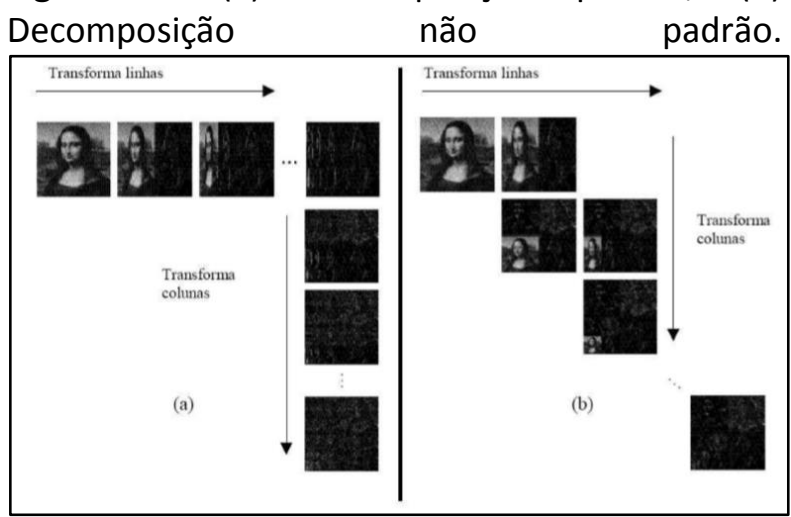

Fonte: (CUPERTINO, 2002)

A decomposição padrão consiste em aplicar a transformada wavelet 1D em cada vetor de linha dos pixels da imagem, até que seja produzido um único valor médio e os outros coeficientes são detalhes de cada linha.

Em seguida aplica-se a transformada 1D em cada coluna da imagem, os valores nessa decomposição são todos detalhes exceto um que é a média global (ARÊDES, 2009).

A decomposição não-padrão realiza uma passagem em cada linha da imagem, determina as médias e as diferenças para cada vetor de pixels. Após isso a média e a diferença são aplicadas a cada coluna do resultado anterior. 
Segundo ARÊDES (2009) o processo é repetido recursivamente, somente nos quadrantes que contêm os coeficientes de aproximação em ambas direções.

A decomposição não-padrão é aplicada, de forma separada na direção vertical e horizontal da imagem, produzindo uma divisão em quatro sub bandas.

Estas sub bandas são definidas como: (LL) nível de aproximação da imagem original, $(\mathrm{HL})$ nível de detalhe horizontal, (LH) detalhe vertical e $(\mathrm{HH})$ detalhe diagonal. Na figura 2 é apresentada de forma esquemática a decomposição não padrão aplicada em dois níveis.

Figura 2. Decomposição não-padrão em 2 níveis.

\begin{tabular}{|c|c|c|}
\hline LL & HL & \multirow{2}{*}{$\mathrm{HL}$} \\
\hline LH & $\mathrm{HH}$ & \\
\hline & & \\
\cline { 1 - 1 } & $\mathrm{HH}$ \\
\hline
\end{tabular}

\subsubsection{Thresholding}

O thresholding é a etapa onde são removidos os ruídos da imagem por meio do uso dos coeficientes wavelet. Para realização de tal tarefa são necessários os seguintes itens: (ARÊDES, 2009)
- $\quad \delta($.$) função de thresholding ou$ shrinkage;

- $\quad \check{\sigma}$ estimativa de desvio padrão do ruído;

- $\lambda$ regra de escolha do valor de thresholding.

A função de thresholding ou shrinkage determina a forma de aplicação do threshold nos coeficientes; as mais utilizadas são hard e soft thresholding, que podem ser vistas na Figura 3. (ARÊDES, 2009).

Figura 3. Comparação entre o Hard e Soft Thresholding.
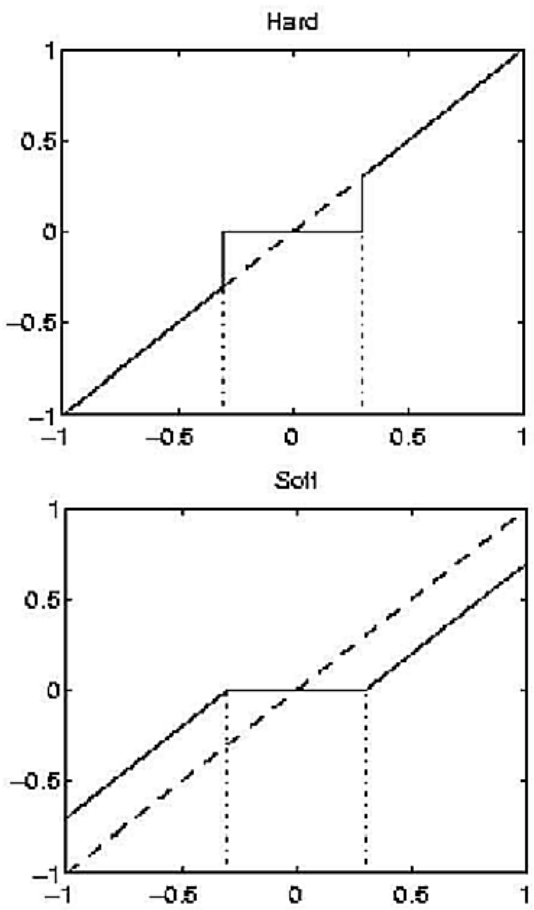

Fonte: (ARÊDES, 2009 apud THE MATHWORKS Inc., 2006)

O hard thresholding consiste em manter ou zerar os coeficientes wavelets. Se o valor absoluto do coeficiente é maior que o threshold, ele é mantido caso contrário é anulado. A função do hard thresholding é 
dada pela Equação 4 (FODOR; KAMATH, 1996).

$$
\delta_{\lambda}^{H}(w)=w I_{\{|w| \geq \lambda\}}
$$

O soft thresholding consiste em ajustar ou zerar os coeficientes wavelet. Se o valor absoluto do coeficiente é maior que o threshold ele é ajustado para um valor prédefinido de thresholding $\lambda$. Caso contrário ele é zerado, a função do soft thresholding é dada pela Equação 5 (FODOR; KAMATH, 1996).

$$
\delta_{\lambda}^{S}=\operatorname{sgn}(w)(|w|-\lambda) I_{\{|w| \geq \lambda\}}
$$

O método utilizado para determinar o valor do threshold, é conhecido como thresholding universal que é dado pela Equação 6 (DONOHO; JONHSTONE, 1994).

$$
\lambda_{U N I}=\hat{\sigma} \sqrt{2 \log N}
$$

Onde $N$ é o tamanho do sinal e $\hat{\sigma}$ a variância, que não é conhecida e deve ter seu valor estimado por meio da técnica de Shrinkage MAD (DENGWEN; WENGANG, 2008). Tal técnica é baseada no desvio médio absoluto dos coeficientes wavelet, e é dada pela seguinte equação:

$$
\hat{\sigma}=\frac{M A D(|w|)}{0.6745}
$$

Onde $w$ é o coeficiente wavelet e $M A D$ é o desvio médio absoluto (Equação 8).

$$
M A D=\frac{\sum|x-\bar{X}|}{n}
$$

\subsection{Trabalhos Relacionados}

Métodos de identificação de dispositivos geradores de imagens foram propostos por vários autores, estes utilizaram diferentes abordagens, como defeitos em pixels e ruído introduzido (GERADTS et al., 2001).

A abordagem proposta por Khanna, Mikkilineni e Delp (2009), utiliza o padrão de referência do scanner. $O$ processo de digitalização possui a característica de ser linear, capturando a imagem por partes. $\mathrm{O}$ padrão ou assinatura do scanner é obtido realizando a média do ruído linear de diversas imagens provenientes de uma mesma fonte.

A extração do ruído se dá com o uso de um filtro baseado em wavelet. O processo de identificação é feito a partir da correlação da assinatura do scanner, e da imagem para qual se deseja saber a origem (KHANNA; MIKKILINENI; DELP, 2009).

Uma das desvantagens desta abordagem é que a imagem investigada deve ser digitalizada de modo bastante semelhante as imagens utilizadas na etapa de treinamento.

Para contornar este impasse Khanna, Mikkilineni e Delp (2009), utilizaram medidas estatísticas dos vetores de ruído, e classificadores de padrão. O método proposto obteve acurácia em torno de $90 \%$. 
Devido a semelhança de funcionamento entre scanners e câmeras digitais os métodos de identificação podem ser expandidos entre os dispositivos.

Uma abordagem é utilizar as imperfeições do sensor de imagem. Lukáš, Fridrich e Goljan (2005) descrevem um método em que são analisadas as nãouniformidades nos pixels da imagem que podem denotar defeitos no sensor, danos à lente ou poeira acumulada, por exemplo.

A partir da média de várias imagens, um filtro baseado em wavelet, é aplicado e a assinatura da câmera é extraída. Após esta etapa é realizada a correlação entre os pixels não uniformes do padrão, com a imagem investigada.

O método proposto por Lukáš, Fridrich e Goljan (2005) atingiu em seus experimentos a acurácia $100 \%$ quando deveria distinguir entre dois modelos de câmeras.

\section{METODOLOGIA}

\subsection{Criação da Base de Imagens}

A base de imagens foi criada com 0 uso de 80 imagens de diferentes tipos, como páginas de revista, apresentação de slides, trechos de código, cartolina preta e principalmente documentos, como pode ser visto na Figura 4.

O processo de digitalização envolveu três scanners. Os modelos utilizados compõem impressoras multifuncionais de diferentes modelos e ano de fabricação, os dispositivos usados foram: (i) HP Photosmart C3180, (ii) HP PSC 1410 e (iii) HP Deskjet 2050.

Para a digitalização foi utilizado o software Fax e Scanner do Windows, com a resolução de 300 DPI e o formato de imagem TIFF, gerando imagens com tamanho aproximado de $3 \mathrm{MB}$.

Figura 4. Exemplo de imagens da base.

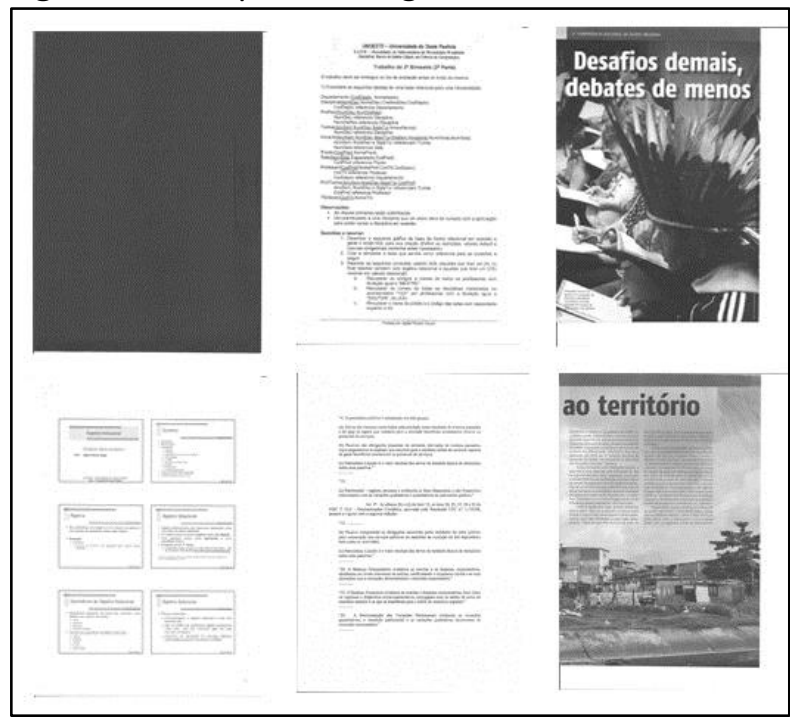

\subsection{Implementação}

A ferramenta é constituída por três módulos, sendo eles: (i) filtro de ruídos, (ii) processador de ruído, (ii) identificador de scanner.

O filtro de ruído é resultado da implementação da transformada wavelet de Haar, das técnicas thresholding e de um filtro de luminância, para a conversão em tons de cinza. Para aplicar a DWT é necessário que seja informado quantos níveis se deseja utilizar. 
E nos testes foi empregado apenas um nível; após utilizou-se o thresholding, onde existe a opção de duas regras soft e hard, esta filtragem é aplicada somente nas sub bandas $\mathrm{HL}$, $\mathrm{LH}$ e $\mathrm{HH}$ em que os coeficientes representam informação de alta frequência.

O processador de ruídos compreende determinados algoritmos que visam refinar o ruído obtido após a etapa de filtragem e estes algoritmos são: Otsu, threshold manual, média, dilatação, erosão, inversão de cores e remover borda.

O Otsu e threshold manual, evidenciam apenas o ruído mais importante. A média é aplicada em uma janela $3 \times 3$, onde caso o pixel esteja abaixo dela é anulado e se estiver acima será mantido.

A dilatação e a erosão são utilizadas para expandir ou reduzir o ruído. A inversão de cores é útil quando a média não possuí um resultado satisfatório, evidenciando assim o ruído existente.

Já a remoção de bordas, elimina o ruído desnecessário gerado nas bordas da imagem, e o usuário define o quanto remover.

O identificador de scanner recebe uma imagem contendo os pontos que foram identificados como as características únicas do scanner; e com o uso dela ele realiza comparações através de subtrações dos pixels encontrados para assim determinar a origem do scanner.

\subsection{Identificador de Scanners}

Tendo como entrada uma imagem contendo os pontos que foram identificados como a "impressão digital do scanner", o que pode ser visto por exemplo na figura 5, é dado início ao processo de identificação.

Figura 5. Ruído de um scanner

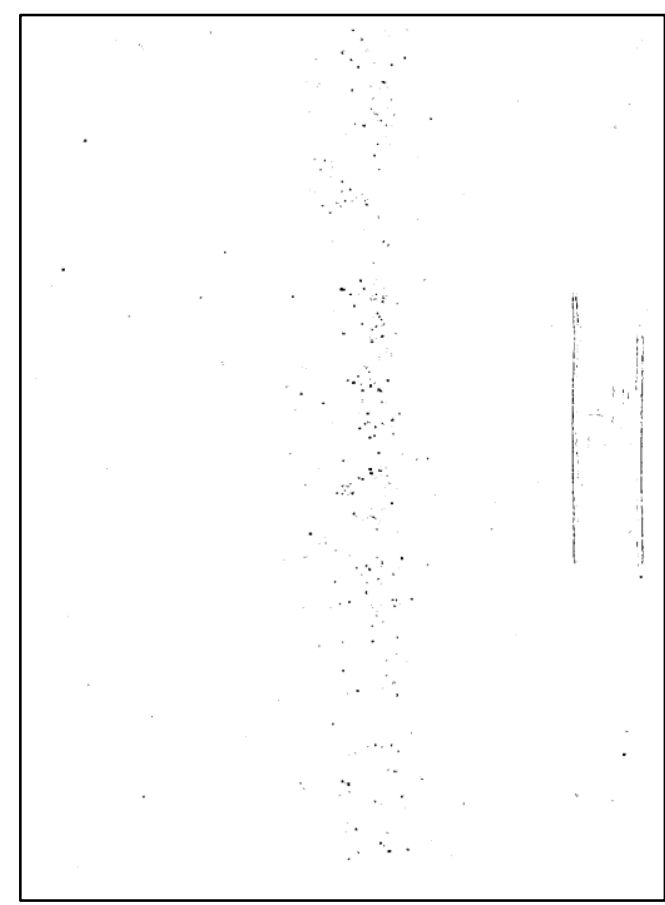

Primeiramente indica-se onde está a imagem que foi utilizada para a extração do ruído, após isto obtêm-se as coordenadas dos pixels correspondentes ao ruído, em seguida indica-se onde será salvo o arquivo .CSV contendo os resultados da comparação, e por fim a pasta contendo as imagens e a quantidade que serão utilizadas no processo; concluídos estes passos realiza-se a identificação. 


\subsubsection{Processo de Identificação}

Para distinguir um scanner obtêm-se o valor do pixel $\left(p_{1}\right)$ na imagem utilizada para a extração do ruído, em outra imagem na mesma coordenada também se obtêm o valor do pixel $\left(p_{2}\right)$.

Com estes é realizada uma subtração onde é considerado apenas o valor absoluto, e o resultado compõe um somatório, como visto na equação 9.

$$
\sum_{1}^{n}\left|p_{1}-p_{2}\right|
$$

Após a conclusão são obtidas várias medidas estatísticas para cada canal (RGB), estas são: média das subtrações, mediana, desvio padrão, máximo e mínimo, também é feita a média das médias das subtrações, obtendo assim um valor único, não sendo necessário analisar os canais separadamente.

Terminado o processo o mesmo deve ser feito novamente, apenas trocando a pasta que contém as imagens e criando um novo arquivo para os resultados.

Concluído o processo de se obter dados através das subtrações, é necessária a análise dos dados, para enfim identificar a origem das imagens.

A análise deve ser feita comparando o valor da média das médias das subtrações de cada canal, obtidos em todos os testes realizados com a mesma imagem de extração de ruído, onde a média que possui o menor valor indica o scanner de origem da imagem.

\section{EXPERIMENTOS}

Os testes realizados utilizaram três scanners dos seguintes modelos HP Photosmart C3180, HP PSC 1410 e HP Deskjet 2050.

O processo de obtenção dos padrões de ruído de cada dispositivo pode ser visto na figura 6.

Figura 6. Fluxograma de Obtenção do Ruído Assinatura.

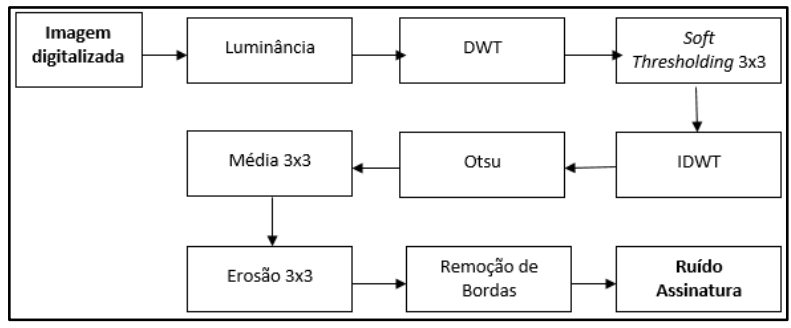

Com os padrões extraídos (Figura 7), os testes realizados utilizaram 80 imagens para cada scanner e estas provenientes da base de imagens que foi criada. 
Figura 7. Padrões de Ruído Obtidos

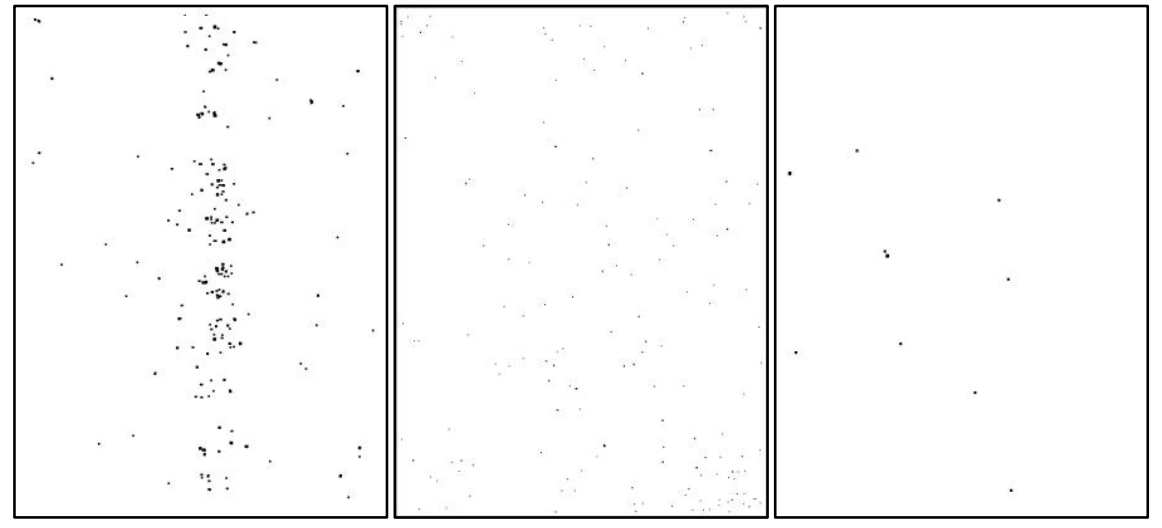

Os resultados dos testes encontramse na Tabela 1; analisando-os é possível verificar que foram satisfatórios quanto a identificação.

A acurácia média foi de 87,5\%; o scanner C3180 conseguiu uma taxa menor de acertos, e isso se deve a um fato que foi observado durante a realização do projeto. Quando há muito ruído, a identificação é prejudicada, pois se torna necessário o uso de muitos pixels no processo; este ruído em geral é principalmente relacionado à idade do dispositivo.

Tabela 1. Resultados dos Testes

\begin{tabular}{|c|c|c|c|}
\cline { 2 - 4 } \multicolumn{1}{c|}{} & $\mathbf{C 3 1 8 0}$ & $\mathbf{2 0 5 0}$ & $\mathbf{1 4 1 0}$ \\
\hline $\mathbf{C 3 1 8 0}$ & $73,75 \%$ & $0 \%$ & $26,25 \%$ \\
\hline $\mathbf{2 0 5 0}$ & $1,25 \%$ & $98,75 \%$ & $0 \%$ \\
\hline $\mathbf{1 4 1 0}$ & $10 \%$ & $0 \%$ & $90 \%$ \\
\hline
\end{tabular}

\section{CONSIDERAÇÕES FINAIS}

De maneira geral verifica-se que o método desenvolvido funciona e é capaz de identificar satisfatoriamente diferentes scanners.

É relevante observar que a acurácia média de $87,5 \%$, foi obtida sem o uso de métodos de aprendizagem de máquina, reduzindo assim a sua complexidade.

Um fator a se considerar quanto a finalidade da ferramenta é a degradação imposta pelo tempo ao equipamento, pois com o passar dos anos o nível de ruído tende a aumentar dificultar assim a identificação de imagens/documentos digitalizados em épocas passadas.

Porém, com um refinamento do método é possível melhorar a acurácia e assim como contornar este problema.

\section{REFERÊNCIAS}

ARÊDES, B. A. R. Técnicas de Wavelet Thresholding aplicadas no processo de denoising de imagens digitais. 2009. $106 \mathrm{f}$. Dissertação (Mestrado) - Pontifícia 
Universidade Católica de Minas Gerais, Belo Horizonte, 2009.

CUPERTINO, P. Wavelets: uma introdução. Sociedade Brasileira de Matemática, Rio de Janeiro, v. 33, 2002.

DENGWEN, Z.; WENGANG, C. Image denoising with an optimal threshold and neighbouring window. Pattern Recognition Letters, n. 29, 2008.

DONOHO, D. L.; JONHSTONE, I. M. Ideal Spatial Adaptation via Wavelet Shrinkage. Biometrika, v. 81, 1994.

FARIA, R. R. A. Aplicação de Wavelets na análise de gestos musicais em timbre de instrumentos acústicos tradicionais. 1997. Dissertação (Mestrado) - Escola Politécnica do Estado de São Paulo, 1997.

FODOR, I. K.; KAMATH, C. On denoising images using Wavelet-Based statistical techniques. Livermore, CA.: Center for Applied Scientific Computing Lawrence Livermore national Laboratory, 1996.

GERADTS, Z. J. et al. Methods for identification of images acquired with digital cameras. Enabling Technologies for Law Enforcement and Security, v. 4232, n. 1, p. 502-512, 2001.

GOMES, J. M. A Forense computacional e os discos de estado sólido. In: INTERNATIONAL CONFERENCE ON FORENSIC COMPUTER SCIENCE - ICOFCS 2012, 7. Proceeding... Brasília, ABEAT, 2012. p. 07. https://doi.org/10.5769/C2012001

KHANNA, N.; MIKKILINENI, A.; DELP, E. Scanner identification using feature-based processing and analysis. IEEE Transactions on Information Forensics and Security, v. 4, mar. 2009. https://doi.org/10.1109/TIFS.2008.2009604

LIMA, P.C. Wavelets: uma introdução. 2003. Disponível

em: <http://www.mat.ufmg.br/ /ima/artigos/rmu 33.pdf> Acesso em: 23 nov. 2015.

LUKÁŠ, J.; FRIDRICH, J.; GOLAN, M. Determining digital image origin using sensor imperfections. In: THE SPIE INTERNATIONAL CONFERENCE ON IMAGE AND VIDEO COMMUNICATIONS AND PROCESSING. Proceedings... v. 5685, n. 1, p. 249-260, 2005.

SANTOS, L. M. R. Segmentação da fala utilizando transformadas Wavelets Discreta DWT. Universidade Luterana no Brasil, 2004. 